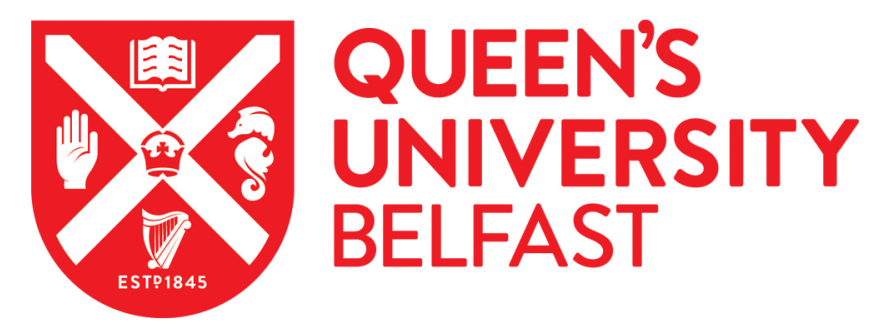

\title{
Spectrum Sharing and Power Allocation Optimised Multi-hop Multi- path D2D Video Delivery in Beyond 5G Networks
}

Tran, Q. N., Vo, N-S., Bui, M. P., Phan, T-M., Nguyen, Q-A., \& Duong, T. Q. (2021). Spectrum Sharing and

Power Allocation Optimised Multi-hop Multi-path D2D Video Delivery in Beyond 5G Networks. IEEE

Transactions on Cognitive Communications and Networking. https://doi.org/10.1109/TCCN.2021.3133838

Published in:

IEEE Transactions on Cognitive Communications and Networking

Document Version:

Peer reviewed version

Queen's University Belfast - Research Portal:

Link to publication record in Queen's University Belfast Research Portal

Publisher rights

Copyright 2021, IEEE

This work is made available online in accordance with the publisher's policies. Please refer to any applicable terms of use of the publisher.

\section{General rights}

Copyright for the publications made accessible via the Queen's University Belfast Research Portal is retained by the author(s) and / or other copyright owners and it is a condition of accessing these publications that users recognise and abide by the legal requirements associated with these rights.

Take down policy

The Research Portal is Queen's institutional repository that provides access to Queen's research output. Every effort has been made to ensure that content in the Research Portal does not infringe any person's rights, or applicable UK laws. If you discover content in the Research Portal that you believe breaches copyright or violates any law, please contact openaccess@qub.ac.uk. 


\title{
Spectrum Sharing and Power Allocation Optimised Multi-hop Multi-path D2D Video Delivery in Beyond 5G Networks
}

\author{
Quang-Nhat Tran, Nguyen-Son Vo*, Minh-Phung Bui, Thanh-Minh Phan, Quynh-Anh Nguyen, \\ and Trung Q. Duong, Fellow, IEEE
}

\begin{abstract}
In this paper, we propose a joint spectrum and power optimisation (SPO) for multi-hop multi-path (MHMP) device-to-device (D2D) video delivery in beyond 5G (B5G/6G) networks, where the system resources such as spectrum, energy, storage, and content of mobile users (MUs), are taken into account. Particularly, the downlink spectrum resources of the sharing users (SUs) are reused by the transmitters (TXs) of D2D hops and the energy resources of the TXs are utilised for D2D communications. We further exploit the videos stored in the caching users (CUs) located more than one D2D hop far away from the requesting users (RUs) to establish MHMP D2D video delivery sessions from the CUs to the RUs. Then, the SPO problem is formulated for the optimal spectrum sharing pairs of SUs and TXs and the optimal transmission powers allocated to the TXs. Genetic algorithms (GAs) are developed to solve the SPO problem with respect to both binary variable (spectrum sharing) and real variable (power allocation). The SPO solution allows the RUs to alternately request the videos not only from the macro base station over conventional cellular networks but also from the CUs over MHMP D2D communications at the highest quality of service, i.e., maximum video delivery capacity and low power consumption. Simulation results are analysed to demonstrate the feasibility of GAs and the benefits of the proposed SPO solution in comparison with other conventional schemes.
\end{abstract}

Keywords-Beyond 5G/6G networks, caching networks, downlink spectrum resource sharing, multi-hop multi-path D2D communications, power allocation, video applications and services.

\section{INTRODUCTION}

It has been a very long way to the launch of $5 \mathrm{G}$ networks that involve all aspects of wireless communications and emerging technologies to provide the end users with enhanced mobile broadband, ultra-reliable low latency communications, and

This paper was presented in part at the 4th International Conference on Recent Advances in Signal Processing, Telecommunications \& Computing (SigTelCom), Hanoi, Vietnam, Aug. 2020.

Quang-Nhat Tran and Minh-Phung Bui are with Van Lang University, Ho Chi Minh City 700000, Vietnam (e-mail: nhat.tq@vlu.edu.vn; phung.bm@vlu.edu.vn).

* Corresponding author: Nguyen-Son Vo is with the Institute of Fundamental and Applied Sciences, Duy Tan University, Ho Chi Minh City, 700000, Vietnam, and also with the Faculty of Electrical-Electronic Engineering, Duy Tan University, Da Nang, 550000, Vietnam (e-mail: vonguyenson@duytan.edu.vn).

Thanh-Minh Phan and Quynh-Anh Nguyen are with Ho Chi Minh City University of Transport, Ho Chi Minh City 700000, Vietnam, (e-mail: minh.phan@ut.edu.vn; quynhanh.nguyen@ut.edu.vn).

Trung Q. Duong is with Queen's University Belfast, Belfast, U.K, (e-mail: trung.q.duong@qub.ac.uk). massive machine type communications [1]. However, it is more challenging by 2023 that the number of connections in beyond $5 \mathrm{G}(\mathrm{B} 5 \mathrm{G} / 6 \mathrm{G})$ networks will grow over 100 -fold to reach 1.4 billion, from about 13 million in 2019 [2]. In this context, B5G/6G networks have been started studying immediately to propose more disruptive solutions and technologies that can catch up with the ever-proliferation of dense mobile users (MUs) [3], especially the ones that request various video applications and services (VASs) at extremely high data rate and quality of service (QoS).

The proliferation of dense MUs requesting the VASs in B5G/6G networks poses a set of challenges to the Internet service providers (ISPs) and the content providers (CPs). The VASs, e.g., virtual reality, e-health and education, public safety surveillance and management, entertainment etc., consume a huge amount of spectrum, energy, and storage resources which are scarce and limited in mobile cellular networks [4]-[6]. To meet the benefits of all the ISPs, CPs, mobile cellular network carriers, and MUs, on the one hand, it is necessary to propose new network architecture modifications and upgrades. On the other hand, B5G/6G networks have placed a higher priority on soft and intelligent solutions without any architecture changes for low cost deployment [7]. This in turn stimulates the need for designing a proper resource-efficient solution, i.e., a better spectrum, energy, and storage management for VASs in $\mathrm{B} 5 \mathrm{G} / 6 \mathrm{G}$ networks.

One of the most efficient solutions is utilising the resources of MUs that have available spectrum and energy resources for sharing and storage resource to cache the videos. This way can establish multiple video delivery sessions over direct unicast or multicast device-to-device (D2D) communications [8]-[35]. In other words, the videos are offloaded to the requesting users (RUs) in the vicinity of transmissions at high capacity, and thus mitigating the traffic congestion at the backhaul links of the macro base stations (MBSs). The limit of direct D2D communications is that there exist many videos cached in dense MUs, namely caching users (CUs), located more than one D2D hop far away from the RUs, but they are not exploited for multi-hop multi-path (MHMP) D2D video delivery.

In fact, the advantage of dense MUs has been studied to provide the RUs with an alternately efficient way to receive the videos from the CUs over MHMP D2D communications (including direct D2D communications) besides from the MBSs over common cellular communications [36]-[41]. The problem of these studies is that they have not utilised the 
MUs' behavior represented by the popularity of the videos cached in the CUs to further satisfy the RUs. There have had some studies on MHMP D2D communications that are suitable for VASs since they consider the cached videos in the CUs and/or the popularity of videos [42]-[49]. However, the aforementioned studies have not applied a joint spectrum and power optimisation (SPO) that allows the RUs to flexibly receive the videos over the MHMP D2D communications and/or the MBSs at the best QoS, i.e., the highest video delivery capacity and low transmission power consumption.

In this paper, we propose the SPO solution for VASs over MHMP D2D communications in B5G/6G networks. This is an extended solution from our previous work, namely spectrum sharing optimisation (SSO) [50]. Particularly, in SSO solution, we have considered the storage resource to cache the videos in the CUs and the downlink spectrum resource of the sharing users (SUs) for D2D communications, so as to establish multiple video delivery sessions over MHMP D2D communications from the CUs to the RUs. The main contributions of this paper are summarised as follows:

- We consider the energy resource to allocate the transmission powers to the transmitters (TXs) of D2D hops used for forwarding the requested videos. This way can simultaneously improve the video delivery capacity and conserve the total transmission power of the TXs.

- We formulate the SPO problem and then solve it for the optimal downlink spectrum sharing and power allocation. The SPO solution fully utilises the downlink spectrum resources of the SUs by enabling an arbitrary SU to share its downlink spectrum resource to multiple TXs for D2D communications in different paths from the CUs to the RUs. It also takes into account the popularity of videos to serve the RUs more efficiently and a target signal to interference plus noise ratio (SINR) of the SUs to limit the interferences caused by the transmissions of the TXs that reuse the same downlink spectrum resources shared by the SUs.

- Genetic algorithms (GAs), which belong to a family of adaptive heuristic searching algorithms based on the evolutionary principles of nature selection and genetic variation, are applied to solving the SPO problem. On the one hand, the benefit of GAs is that they can consider multiple peaks simultaneously without concerning whether the searching space is unimodal or multimodal, but finding globally (exactly or approximately) optimal results at a reasonable and flexible complexity [13], [51]-[53]. Meanwhile, exhaustive algorithms can find the exact results, but causing computationally expensive and time-consuming, therefore not feasible to be applied to the real-world problems [18], [19]. Other promising methods such as dynamic programming, stochastic and game learning, greedy algorithm, and heuristic strategy, can provide lower complexity, but less accuracy [53]. On the other hand, we develop the conventional GAs, and thus they are able to solve the SPO problem with respect to both binary variable for spectrum sharing and real variable for power allocation, under many complicated constraints, which cannot be done by the aforementioned methods.

- Simulation results are insightfully analysed to demonstrate the feasibility of GAs and the benefits of the proposed SPO solution in comparison with other conventional schemes in terms of average video delivery capacity and total transmission power consumption. And importantly, in SPO solution, the RUs can be served with the best QoS of VASs cooperatively from the CUs over MHMP D2D communications and/or from the MBS over common cellular communications.

The rest of this paper is organized as follows. In Section II, we review the related works on D2D communications including unicast, multicast, and MHMP D2D communications. Section III introduces a system model of VASs over MHMP D2D communications in B5G/6G networks and describes how it works. Then, we formulate the proposed system in Section IV that consequently allows us to propose the SPO problem and solution with GAs in Section V. Section VI is dedicated to analysing the simulation results to demonstrate the performance of GAs and SPO solution in comparison with other conventional schemes. Finally, we conclude the paper in Section VII.

\section{RELATED WORKS}

In this section, we provide more discussions on the related works to highlight the new contributions of our paper compared with previous works. The related works include unicast D2D communications [8]-[14], [16]-[21], multicast D2D communications [22]-[35], and MHMP D2D communications [36]-[49], presented in the sequel.

\section{A. Unicast D2D Communications}

Utilising D2D communications to offload the contents from MU to MU directly is one of the most efficient solutions to improve the QoS (capacity, coverage, connectivity, and delay) and spectrum- and energy-efficiency, while mitigating the traffic congestion at the backhaul links of both MBSs and smallcell base stations (SBSs) [8]-[14], [16]-[21]. In particular, the feasibility of D2D networks was investigated with respect to the transmission power distributions of the TXs to achieve different target SINRs [8]. It is better if clustering technique [9] is deployed to manage the interference, i.e., preventing from the outage probability of D2D communications, and then applying power control scheme [10]. Similarly, in [11], downlink spectrum allocation is deployed to guarantee the QoS fairness in the first stage and power control scheme is applied to maximise the utility of MUs in the second stage.

In [12], the authors proposed an MUs and files clustering scheme to reduce the complexity of D2D communications with caching. The MUs, who have the same interest of a specific content (e.g., sport, education, latest films, etc.), are put into a cluster. In this way, the download rate is improved by enabling multiple CUs to collaboratively send the same file to an RU. The work in [13] further considered encoding the requested video into multiple descriptions and collaborating between the MBS and the CUs in sending the descriptions to the RU at high quality of experience (QoE) and low energy consumption. 
In terms of system model analysis, the authors in [14] proposed a cooperative spectrum sharing scheme in which direct D2D communications and two-way amplify-and-forward relaying communications are combined to enhance the system performance. In [16], an interesting D2D communications system in heterogeneous networks was proposed in the presence of interferences caused by the coexistence of MBS, SBSs such as pico-cell base stations, and femto-cell base stations (FBSs), and TXs of other D2D communications. Then, the spectrum efficiency of D2D communications was investigated by finding the optimal transmission powers allocated to the TXs. To make D2D communications with caching become more efficient, the optimal content placement in D2D networks is found in accordance with the mobility of MUs to minimise the workload at the backhaul link of the MBS [17].

In [18], [19], D2D communications with caching were exploited to mitigate the workloads at not only the MBS but also the FBSs. These works consider the social relationship between the MUs [18] and the video encoding rates (video versions with different qualities) [19] to enhance the QoS and the caching performance. When D2D communications with caching play an important role in a large scale of B5G/6G networks, a D2D caching system should be studied to gain an excellent caching performance [20]. To do so, the hit ratio is divided into six parts depending on the offloading status (selfoffloading and D2D offloading), preferences of MUs (common MUs and individual MUs), and patterns of contents (little temporal changes and large temporal changes). Consequently, there also have six caching areas of the CUs to obtain an accurate caching strategy that can satisfy the diverse demands of the RUs. Meanwhile, the work in [21] has only focused on the distribution of individual MUs' content preference. To this end, the caching efficiency is maximised with respect to the quality and quantity recommendations of individual MUs and the caching capacity requirements.

We can see that the aforementioned studies have exploited the spectrum, energy, storage, and content resources of MUs and the characteristics of contents, to propose various models and techniques of D2D communications with caching. However, such D2D communications work in unicast transmission mode that cannot utilise the benefit of nature of wireless environment for broadcasting the contents. In addition, a huge amount of the MUs' resources, which can be available around but more than one D2D hop far away from the RUs, have not been utilised yet. In this paper, these resources are fully taken into account in the SPO solution so that the B5G/6G networks with MHMP D2D communications assisted are established to serve the RUs the highest QoS of VASs.

\section{B. Multicast D2D Communications}

The performance of D2D communications with caching can be significantly improved if further considering the broadcast nature of wireless environment and the fact that there are many RUs in the vicinity of each other having the same interest of contents. Therefore, multicast D2D communications have been studied to provide the RUs with a higher QoS of VASs [22]-[35]. The most important technique that is prerequisite for multicast D2D communications with caching is clustering the RUs who are interested in the same contents. In each cluster, a cluster head $(\mathrm{CH})$ is elected to provide the others with a lower transmission delay [22] and the best energy-quality efficiency [23]. By following a specific clustered distribution model and an agglomerative hierarchical clustering algorithm, the joint optimal downlink spectrum and power allocation solutions were studied to maximise the system sum rate [24] and further improve the energy efficiency [25], [26]. The clustering and uplink spectrum sharing can be combined to improve the resource efficiency as studied in [27], meanwhile only selecting the dynamic links was used for mitigating the interference and thus obtaining the nash equilibrium points [28].

In [29]-[31], multicast techniques can be applied to the MBSs and/or FBSs besides D2D communications to propose flexible VASs in B5G/6G networks. After clustering, the social relationship between the $\mathrm{CH}$ s and the other RUs in each cluster is defined to improve the QoS. It is crucial that the works [29]-[31] have addressed the problem of unfair QoS among the RUs in different clusters caused by multicast transmissions over diverse characteristics of wireless channels from the $\mathrm{CHs}$ to the RUs. In [32], the RUs can also access the contents flexibly from the MBS, SBSs, and CHs with high capacity and low delay, but multicast technique is only applied to D2D communications. Meanwhile, the system model in [33], which aims to analyse the coverage and the transmission delay, allows the RUs to receive the multicast video delivered from the MBS and the CHs. Without clustering, the authors in [34] have proposed a joint channel and power allocation to maximise the total sum rate of the SUs and RUs by unicasting from the MBS and multicasting from the TXs, respectively. Simply but efficiently, the work [35] only considers selecting a number of minimum caches associated with their social group to satisfy the RUs.

However, the existing problems are that the current studies on multicast D2D communications have not utilised the available energy, storage and content resources of MUs located around but a little bit far away, i.e., more than one D2D hop, from the RUs. Besides, the characteristics of contents and MUs have not been considered to maximise the satisfaction of RUs. Some of these existing problems have been addressed by MHMP D2D communications, which are discussed in more detail below.

\section{MHMP D2D Communications}

In fact, multi-hop D2D communications have been studied in the literature to address the problems of unicast and multicast D2D communications [36]-[41]. However, most of these studies focus on single content delivered from the $\mathrm{CU}$ to the RU to improve the power efficiency by social-aware relay selection scheme [36], the system capacity and energy efficiency by multiple QoE metrics based relay selection scheme with spectrum resource sharing [37], the system utility by mobility- and activeness-aware relay selection scheme with spectrum resource sharing [38], the system capacity by location- and interference-aware relay selection scheme [39], and the energy- and spectrum-efficiency by two-timeslot physical layer network coding with orthogonal sharing 
channels [40]. However, these studies have not utilised the skewed popularity of multiple contents to satisfy the RUs except that the scaling law for more practical case of popularity distribution was analysed in [41]. Furthermore, multi-path D2D communications have not been considered to enhance the satisfaction of the RUs.

In MHMP D2D communications, it is prerequisite to study routing algorithms [39], [42]-[44]. We can see that because MHMP D2D communications introduce a higher opportunity of information disclosed to the eavesdroppers, the routing algorithms often focus on security issues. In [42], the security implications of multi-path routing were investigated to demonstrate that interference disjoint paths can effectively inhibit the eavesdroppers. More complicatedly in [43], the authors combined data slitting and shuffling together with coding mechanisms to prevent the eavesdroppers from having information transmitted by the legal MUs. In addition, the work [44] proposed a low-overhead multi-hop routing algorithm that can reduce the number of control messages exchanged in D2D communications. However, routing is out of the scope of our SPO solution. Alternately, we apply the interference-aware multi-hop path selection for D2D communications given in [39].

Other studies on MHMP D2D communications that lead to a satisfaction of the RUs have been studied in [45], [46]. This study allows the RUs to be served by both inband and outband modes. The system utility and the satisfaction of the RUs are achieved by deploying an adaptive mode selection. In [47]-[49], MHMP D2D communications have been studied to expand the function of VASs and thus further satisfy the RUs. In particular, an energy budget aware routing was proposed for delivering longer MHMP D2D video sessions by scheduling the routes and video traffic to prevent from the low-battery D2D outages [47]. In [48], the authors proposed a joint coding mode (video coding mode) and multi-path selection (underlay or overlay) for video transmission to enhance the perceived QoS with low energy consumption. Interestingly, a joint resource sharing and segment allocation optimisation solution for video delivery over MHMP D2D communications was proposed in [49]. First, the optimal downlink spectrum resource sharing for MHMP D2D communications is found for maximum video delivery capacity. Second, the segments of the requested video are optimally allocated to different paths depending on the capacity of each path and the intra-popularity of each segment so as to maximise the playback quality of the received video. However, the current studies for MHMP D2D communications have not considered proposing a joint downlink spectrum sharing and power allocation optimisation solution that allows the RUs to flexibly access the VASs from the CUs over MHMP D2D communications and/or from the MBS over common cellular communications at the highest video delivery capacity and low total transmission power consumption.

\section{SySTEM MODEL}

In this paper, we propose the system model for VASs over MHMP D2D communications in B5G/6G networks as shown

\section{TABLE I: Notations}

\begin{tabular}{|c|c|}
\hline Symbols & Specifications \\
\hline $\bar{N}$ & Number of SUs \\
\hline$K$ & Number of CUs, paths, or videos \\
\hline$H_{k}$ & Number of D2D hops in the path $k, k=1,2, \ldots, K$ \\
\hline$h_{k}$ & $\begin{array}{l}\text { D2D hop } h_{k} \text { in the path } k \text {, each has a pair of transmitter (TX) } \\
\text { and receiver (RX), } h_{k}=1,2, \ldots, H_{k}\end{array}$ \\
\hline$v_{n, h_{k}}$ & $\begin{array}{l}\text { Spectrum sharing variable to indicate that the SU } n \text { agrees to share } \\
\text { its downlink spectrum resource with the TX of D2D hop } h_{k} \text { in } \\
\text { the path } k\left(v_{n, h_{k}}=1\right) \text { or does not agree }\left(v_{n, h_{k}}=0\right), n= \\
1,2, \ldots, N\end{array}$ \\
\hline$P_{h_{k}}^{\mathrm{TX}}$ & Transmission power variable allocated to the TX of D2D hop $h_{k}$ \\
\hline$W^{\kappa}$ & System bandwidth \\
\hline$N_{0}$ & Power of additive white Gaussian noise \\
\hline & Target SINR of SUs \\
\hline$P^{\mathrm{M}, \mathrm{SU}}$ & Transmission power from the MBS to the SUs \\
\hline$P^{\mathrm{M}, \mathrm{RU}}$ & Transmission power from the MBS to the RU \\
\hline$G^{\mathrm{x}, \mathrm{y}}$ & $\begin{array}{l}\text { Channel gain between } \mathrm{x} \text { and } \mathrm{y}, \mathrm{x} \in\{\mathrm{M}, \mathrm{TX}\} \text { and } \mathrm{y} \in \\
\{\mathrm{RX}, \mathrm{RU}, \mathrm{SU}\} \text {, here } \mathrm{M} \text { stands for MBS }\end{array}$ \\
\hline$\eta$ & Path loss exponent \\
\hline$r_{k}$ & Popularity or access rate of the video $k$ \\
\hline$\alpha$ & Skewed popularity exponent coefficient among different videos \\
\hline
\end{tabular}

in Fig. 1. The notations of the system are listed in Table I. In the system, there are one MBS, $N$ SUs, $K$ CUs in which the $\mathrm{CU} k$ has already cached the video $k, k=1,2, \ldots, K$, and one RU who requests $K$ videos. Thanks to powerful storage capacity, the MBS can cache all the $K$ videos. There exist $K$ paths from $K$ CUs to the RU. The path $k$ consists of $H_{k}$ D2D hops, each D2D hop $h_{k}$ has a transmitter (TX) $h_{k}$ and a receiver $(\mathrm{RX}) h_{k}, h_{k}=1,2, \ldots, H_{k}$. The communications over D2D hops in all paths are established by reusing the downlink spectrum resources shared by the SUs. When the MBS acknowledges that the RU requests for a number of videos, it initiates the following three steps:

- Step 1 - Collecting system information and establishing MHMP D2D communications: The MBS collects the system information, i.e., wireless channel characteristics, to establish $K$ paths from $K$ CUs to the RU by applying the interference-aware multi-hop path selection schemes for MHMP D2D communications [39]. It further collects the information of 1) the SUs who are willing to share their downlink spectrum resources and 2) the videos including video popularity and video caching placement (which CUs have cached the requested videos).

- Step 2 - Formulating and solving the SPO problem: Based on the information collected in the step 1, the MBS formulates the SPO problem. The SPO problem is solved for 1) the optimal downlink spectrum sharing $\left(v_{n, h_{k}}\right)$ to indicate that if the SU $n, n=1,2, \ldots, N$, agrees to share its downlink spectrum resource with the TX $h_{k}$ of the D2D hop $h_{k}$ in the path $k\left(v_{n, h_{k}}\right.$ $=1)$ or does not agree $\left(v_{n, h_{k}}=0\right)$ and 2) the optimal transmission power $\left(P_{h_{k}}\right)$ allocated to the TX $h_{k}$ of the D2D hop $h_{k}$ in the path $k$.

- Step 3 - Delivering the videos to the RU: Depending on the optimal results found in the step 2, the MBS decides to send the requested videos by itself or assigns the CUs to do over MHMP D2D communications so as to maximise the video capacity delivered to the RU and 


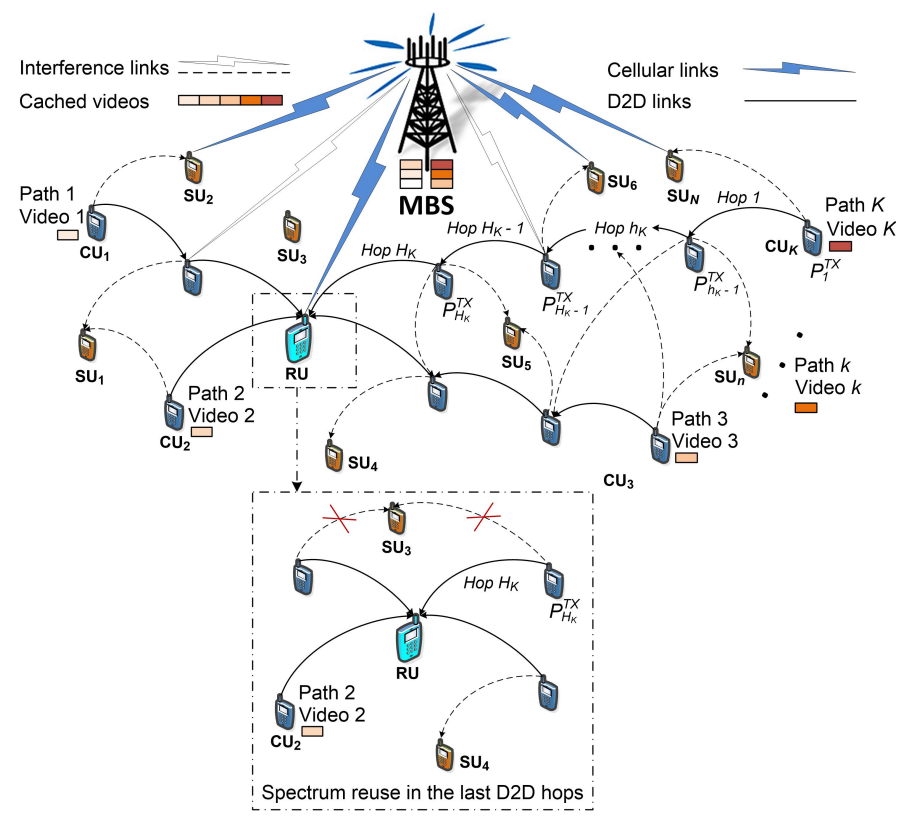

Fig. 1: VASs over MHMP D2D communications in B5G/6G networks.

conserve the total transmission power of the TXs.

It is noted in the step 2 that, in each path, one SU is able to share its downlink spectrum resource with up to one TX for D2D communications, while it can share with up to $K$ TXs in $K$ paths to fully utilise the downlink spectrum resource of the SU. However, more than one TX of the last D2D hops, which are directly connected to the $\mathrm{RU}$, are not allowed to reuse the same downlink spectrum resource shared by one SU (Fig. 1). To do so, the SPO problem considers three constraints related to $v_{n, h_{k}}$ that are 1) $\left.\sum_{h_{k}=1}^{H_{k}} v_{n, h_{k}} \leq 1, n=1,2, \ldots, N, k=1,2, \ldots, K, 2\right)$ $\sum_{n=1}^{N} v_{n, h_{k}} \leq 1, h_{k}=1,2, \ldots, H_{k}, k=1,2, \ldots, K$, and 3$)$ $\sum_{k=1}^{K} v_{n, H_{k}} \leq 1, n=1,2, \ldots, N$. In addition, when the SU $n$ shares its downlink spectrum resource with the TX $h_{k}$, the SINR of the SU $n$ degrades due to the interference caused by the transmission of the TX $h_{k}$. The SPO problem therefore takes the target SINR $\left(\gamma_{0}\right)$ of all the SUs into account to limit the interference impact on themselves. The SPO problem also enables the RU to get the video $k$ from the MBS commonly if 1) an arbitrary D2D hop in the path $k$ is not established because this D2D hop violates the constraint on the target SINR of the SUs or 2) the quality of the channel from the MBS to the RU is better than that from the $\mathrm{CU} k$ to the RU. The proposed system can be easily extended to the context of multiple RUs requesting different sets of videos. In this context, the MBS executes multiple SPO problems to provide all the RUs with the highest QoS.

\section{SySTEM FORMULATIONS}

In this section, to propose the SPO problem and solution, we need to compute the video capacity delivered from the
CUs over MHMP D2D communications and from the MBS to the RU. Then, we compute the objective function, i.e., the average video capacity delivered to the RU, as well as the SINR at the SUs that is used as the constraint in the SPO problem to ensure the given target SINR of the SUs when they share their downlink spectrum resources for MHMP D2D communications.

\section{A. Capacity Delivered to $R U$ from $C U s$}

To compute the video capacity delivered to the RU from the CU $k$ over the path $k$, we have to compute the SINR at the RX $h_{k}$ of the D2D hop $h_{k}, h_{k}=1,2, \ldots, H_{k}$, established by reusing the downlink spectrum resource shared by the SU $n$. This SINR, which is affected by the interference power transmitted from the MBS and the total interference power from the other TXs in the other paths due to reusing the same downlink spectrum resource, is given as below

$$
\gamma_{n, h_{k}}^{\mathrm{RX}}=\frac{v_{n, h_{k}} P_{h_{k}}^{\mathrm{TX}} G_{h_{k}}^{\mathrm{TX}, \mathrm{RX}}}{N_{0}+P^{\mathrm{M}, \mathrm{SU}} G_{h_{k}}^{\mathrm{M}, \mathrm{RX}}+I_{h_{k}}^{\mathrm{RX}}},
$$

where $v_{n, h_{k}}$ is the binary variable to indicate that the SU $n$ agrees to share the downlink spectrum resource with the D2D hop $h_{k}\left(v_{n, h_{k}}=1\right)$ or does not agree $\left(v_{n, h_{k}}=0\right), P_{h_{k}}^{\mathrm{TX}}$ and $G_{h_{k}}^{\mathrm{TX}, \mathrm{RX}}$ are the transmission power from the TX $h_{k}$ to the $\mathrm{RX}^{k} h_{k}$ and the channel gain of the D2D hop $h_{k}, N_{0}$ is the power of additive white Gaussian noise, $P^{\mathrm{M}, \mathrm{SU}}$ and $G_{h,}^{\mathrm{M}, \mathrm{RX}}$ are the transmission power from the MBS to the SU and the channel gain between the MBS and the RX $h_{k}$. The channel gains are modeled as the product of an exponential power fading coefficient with unit mean, i.e., $\sim \exp (1)$, and a standard power law path loss function with pathloss exponent $\eta$ [54]. In addition, $I_{h_{k}}^{\mathrm{RX}}$ is the total interference power caused by the TXs of the D2D hops in the other paths to the RX $h_{k}$ of the D2D hop $h_{k}$ in the path $k$, expressed as

$$
I_{h_{k}}^{\mathrm{RX}}=\sum_{l=1, l \neq k}^{K} \sum_{h_{l}=1}^{H_{l}} v_{n, h_{l}} P_{h_{l}}^{\mathrm{TX}} G_{h_{l}, h_{k}}^{\mathrm{TX}, \mathrm{RX}},
$$

where $P_{h_{l}}^{\mathrm{TX}}$ is the transmission power from the TX $h_{l}$ in the path $l(l \neq k)$ and $G_{h_{l}, h_{k}}^{\mathrm{TX}, \mathrm{RX}}$ is the channel gain between the $\mathrm{TX} h_{l}$ in the path $l$ and the $\mathrm{RX} h_{k}$ in the path $k$.

It is obvious that in the path $k$, the best D2D hop $h_{k}$ with the highest SINR $\left(\gamma_{h_{k}}^{\mathrm{RX}}\right)$ is the one established by the optimal transmission power of the TX $k$ and the optimal downlink spectrum resource shared by the SUs, we have

$$
\gamma_{h_{k}}^{\mathrm{RX}}=\max \left\{\gamma_{n, h_{k}}^{\mathrm{RX}}, n=1,2, \ldots, N\right\} .
$$

Then, the SINR of the path $k$ is equivalent to that of the worst D2D hop with the lowest $\operatorname{SINR}\left(\gamma_{k}^{\mathrm{RX}}\right)$, expressed as

$$
\gamma_{k}^{\mathrm{RX}}=\min \left\{\gamma_{h_{k}}^{\mathrm{RX}}, h_{k}=1,2, \ldots, H_{k}\right\} .
$$

So, the capacity of the path $k$ for delivering the video $k$ from the $\mathrm{CU} k$ to the $\mathrm{RU}$ is given by

$$
C_{k}^{\mathrm{RU}}=W \log _{2}\left(1+\gamma_{k}^{\mathrm{RX}}\right),
$$

where $W$ is the system bandwidth. 


\section{B. Capacity Delivered to $R U$ from MBS}

The proposed SPO solution also enables the RU to receive the video $k$ from the MBS over common cellular communications. This happens if 1) at least one D2D hop in the path $k$ is not shared the downlink spectrum resource by any SUs leading to the result that the path $k$ is not established or 2) the capacity of the path $k$ computed in Eq. (5) is lower than the capacity provided by the MBS. In this case, the signal-tonoise ratio (SNR) and the capacity at the RU delivered from the MBS are respectively expressed as

$$
\gamma_{\mathrm{M}}^{\mathrm{RU}}=\frac{P^{\mathrm{M}, \mathrm{RU}} G^{\mathrm{M}, \mathrm{RU}}}{N_{0}}
$$

and

$$
C_{\mathrm{M}}^{\mathrm{RU}}=W \log _{2}\left(1+\gamma_{\mathrm{M}}^{\mathrm{RU}}\right),
$$

where $P^{\mathrm{M}, \mathrm{RU}}$ is the transmission power from the MBS to the $\mathrm{RU}$ and $G^{\mathrm{M}, \mathrm{RU}}$ is the channel gain between the MBS and the RU.

\section{Average Video Capacity Delivered to $R U$}

So far, the average capacity of all requested videos delivered from the CUs or the MBS to the RU, which is considered as the objective function of the SPO problem, is given by

$$
\bar{C}=\sum_{k=1}^{K} r_{k}\left(\max \left\{C_{k}^{\mathrm{RU}}, C_{\mathrm{M}}^{\mathrm{RU}}\right\}\right),
$$

where $r_{k}$ is the popularity of the video $k$ depending on the MUs' behavior. In this paper, the video popularity is modelled by following Zipf-like distribution [55] given by

$$
r_{k}=\frac{k^{-\alpha}}{\sum_{k=1}^{K} k^{-\alpha}}
$$

here $\alpha$ is the exponent coefficient used to reflect the skewed popularity among different videos, i.e., the higher value of $\alpha$ causes the higher skewed popularity, meanwhile all the videos have the same popularity if $\alpha=0$. We consider the video popularity pattern because it plays an important role in the response of the SPO solution to serve the RU more efficiently.

\section{SINR at SUs}

When the SUs share their downlink spectrum resources with the D2D hops for MHMP communications, the TXs of the D2D hops, which reuse the same downlink spectrum resources with the SUs, transmit the interferences to the SUs, and thus making the SINR at the SUs degraded. In the constraint of the SPO problem, to limit the total interference transmitted by all the TXs impact on the SUs, we compute the SINR at the SU $n$ as below

$$
\gamma_{n}^{\mathrm{SU}}=\frac{P^{\mathrm{M}, \mathrm{SU}} G_{n}^{\mathrm{M}, \mathrm{SU}}}{N_{0}+\sum_{k=1}^{K} \sum_{h_{k}=1}^{H_{k}} v_{n, h_{k}} P_{h_{k}}^{\mathrm{TX}} G_{n, h_{k}}^{\mathrm{TX}, \mathrm{SU}}},
$$

where $G_{n}^{\mathrm{M}, \mathrm{SU}}$ and $G_{n, h_{k}}^{\mathrm{TX}, \mathrm{SU}}$ are the channel gains from the MBS and the TX $h_{k}$ to the SU $n$ [54].

Given the target SINR $\left(\gamma_{0}\right)$ to guarantee the SINR at the SU $n$, each SU can share its downlink spectrum resource with up to $K$ D2D hops in $K$ paths as long as $\gamma_{n}^{\mathrm{SU}} \geq \gamma_{0}$ holds.

\section{SPO PRoblem and GAs Solution}

\section{A. SPO Problem}

The SPO problem is formulated based on 1) the objective function given in Eq. (8) and 2) the constraints on $v_{n, h_{k}}$ to fully exploit the downlink spectrum resources shared by the SUs as discussed in Section III, target SINR $\left(\gamma_{0}\right)$ applied to Eq. (10) to guarantee the SINR of the SUs under the interference impact, and $P^{*}$ to limit the total transmission power of all the TXs. Mathematically, the SPO problem is formulated as below

$$
\begin{gathered}
\max _{v_{n, h_{k}}, P_{h_{k}}^{\mathrm{TX}}} \bar{C} \\
\text { s.t. } \sum_{h_{k}=1}^{H_{k}} v_{n, h_{k}} \leq 1, n=1,2, \ldots, N, k=1,2, \ldots, K \\
\sum_{n=1}^{N} v_{n, h_{k}} \leq 1, h_{k}=1,2, \ldots, H_{k}, k=1,2, \ldots, K \\
\sum_{k=1}^{K} v_{n, H_{k}} \leq 1, n=1,2, \ldots, N, \\
\sum_{k=1}^{K} \sum_{h_{k}=1}^{H_{k}} v_{n, h_{k}} P_{h_{k}}^{\mathrm{TX}} G_{n, h_{k}}^{\mathrm{TX}, \mathrm{SU}} \leq \frac{P^{\mathrm{M}, \mathrm{SU}} G_{n}^{\mathrm{M}, \mathrm{SU}}}{\gamma_{0}}-N_{0}, \\
n=1,2, \ldots, N, \\
P_{h_{k}}^{\mathrm{TX}} \geq 0, k=1,2, \ldots, K, h_{k}=1,2, \ldots, H_{k}, \\
\sum_{k=1}^{K} \sum_{h_{k}=1}^{H_{k}} P_{h_{k}}^{\mathrm{TX}} \leq P^{*},
\end{gathered}
$$

where the constraints (11b) and (11c) are to ensure that one $\mathrm{SU}$ is able to share its downlink spectrum resource with up to $K$ D2D hops in $K$ paths, but with up to only one D2D hop in each path. Meanwhile, the constraint (11d) is to further ensure that one SU cannot share with more than one TX in all the last hops of $K$ paths. This is to prevent from the fact that the RU receives more than one video from its adjacent TXs reusing the same downlink spectrum resource. The constraint (11e) is to guarantee the SINR of the SUs, deriving from Eq. (10) by letting $\gamma_{n}^{\mathrm{SU}} \geq \gamma_{0}$. In addition, the constraints (11f) and (11g) are used to limit the total transmission power consumption of all the TXs.

\section{B. GAs Solution}

In this paper, we apply genetic algorithms (GAs) [56] to solve the above constrained SPO problem. The benefit is that the GAs can provide an exact or approximated global optimal solution within a reasonable memory and time complexity [13], [51]-[53]. However, there are two issues required to address the SPO problem when using the original design of GAs including 1) GAs support simple constraints in the form of lower bound and upper bound, e.g., the constraint (11f), but do not support more complicated constraints like (11b), (11c), (11d), (11e), and (11g) and 2) GAs normally work with real variable [13], [51], [52] or binary variable [53], but the SPO problem has both binary and real variables. 
To address the first issue, we use penalty method [57] to convert the constrained SPO problem to an unconstrained one. To do so, we rewrite the complicated constraints (11b), (11c), (11d), (11e), and (11g) in the following form

$$
\left\{\begin{array}{l}
\Delta v_{n, k}=1-\sum_{h_{k}=1}^{H_{k}} v_{n, h_{k}} \geq 0, n=1,2, \ldots, N, \\
k=1,2, \ldots, K, \\
\Delta v_{k, h_{k}}=1-\sum_{n=1}^{N} v_{n, h_{k}} \geq 0, k=1,2, \ldots, K, \\
h_{k}=1,2, \ldots, H_{k}, \\
\Delta v_{n}=1-\sum_{k}^{K} v_{n, H_{k}} \geq 0, n=1,2, \ldots, N, \\
\Delta \gamma_{n}=\frac{P^{\mathrm{M}, \mathrm{SU}} G_{n}^{\mathrm{M}, \mathrm{SU}}}{\gamma_{0}}-N_{0}- \\
\sum_{k=1}^{K} \sum_{h_{k}=1}^{H_{k}} v_{n, h_{k}} P_{h_{k}}^{\mathrm{TX}} G_{n, h_{k}}^{\mathrm{TX}} \geq 0, n=1,2, \ldots, N, \\
\Delta P=P^{*}-\sum_{k=1}^{K} \sum_{h_{k}=1}^{H_{k}} P_{h_{k}}^{\mathrm{TX}} \geq 0 .
\end{array}\right.
$$

We then derive the penalty function as

$$
\begin{aligned}
F= & \lambda_{1} \sum_{n=1}^{N} \sum_{k=1}^{K}\left(\min \left\{0, \Delta v_{n, k}\right\}\right)^{2}+ \\
& \lambda_{2} \sum_{k=1}^{K} \sum_{h_{k}=1}^{H_{k}}\left(\min \left\{0, \Delta v_{k, h_{k}}\right\}\right)^{2}+ \\
& \lambda_{3} \sum_{n=1}^{N}\left(\min \left\{0, \Delta v_{n}\right\}\right)^{2}+ \\
& \lambda_{4} \sum_{n=1}^{N}\left(\min \left\{0, \Delta \gamma_{n}\right\}\right)^{2}+\lambda_{5}(\min \{0, \Delta P\})^{2},
\end{aligned}
$$

where $\lambda_{1}, \lambda_{2}, \lambda_{3}, \lambda_{4}$, and $\lambda_{5}$ are the constraint violation degrees. The individuals in the current generation are punished by violation degrees if they do not obey the constraints.

Finally, the GAs are capable of solving the unconstrained SPO problem given by

$$
\max _{v_{n, h_{k}}, P_{h_{k}}^{\mathrm{TX}}} \bar{C}_{F}=\bar{C}-F .
$$

It can be seen in (14) that in regard to the penalty function (13), the individuals, which violate the constraints, make the objective function $\bar{C}$ (11a) decreased a punishment amount of $F$ (13). After each generation, the punishment amount gets closer to zero $(F=0)$, i.e., no more individuals violate the constraints, while $\bar{C}_{F}$ becomes maximum simultaneously, and thus the GAs can be terminated.

To address the second issue, each chromosome, which is a binary string to represent an individual, is divided into two substrings. The left sub-strings standing for the binary variables $\left(v_{n, h_{k}}\right)$ are directly passed on to the fitness function evaluation (i.e., computing $\bar{C}_{F}$ ) for ranking, meanwhile the right substrings must be converted to real variables $\left(P_{h_{k}}^{\mathrm{TX}}\right)$ by using a binary string to real variable conversion operator (b2r) [56] before passing on to the fitness function evaluation for ranking. The detailed GAs for solving the unconstrained SPO problem are presented in Algorithm 1.

In Algorithm 1, TC is the termination conditions of GAs. The GAs terminate if satisfying one of the two following conditions: 1) $F \leq 10^{-3}$ and $\bar{C}_{F}$ does not change in 10
Algorithm 1 GAs implementation

Input: System parameters listed in Table II

$N_{P}=10,000$ : Number of individuals in the population

$P R E C I_{B}=1: 1$ bit for the precise definition of each binary variable of $v_{n, h_{k}}$

$P R E C I_{R}=100: 100$ bits for the precise definition of each real variable of $P_{h_{k}}^{\mathrm{TX}}$

$B=N \times \sum_{k=1}^{K} H_{k}$ : Number of binary variables of $v_{n, h_{k}}$

$R=\sum_{k=1}^{K} H_{k}$ : Number of real variables of $P_{h_{k}}^{\mathrm{TX}}$

$V_{B}=P \bar{R} E C I_{B} \times B$ : Length of the left substring

$V_{R}=P R E C I_{R} \times R$ : Length of the right substring

$N_{B}=V_{B}+V_{R}$ : Length a chromosome or a binary string representing an individual in the potential solution set $\left\{X_{i}\right\}, i=1,2, \ldots, N_{P}$

$P_{G}=0.9:$ Generation gap

$P_{C}=0.6$ : Crossover probability and $P_{M}=10^{-9}$ : Mutation probability

$\left\{\lambda_{1,2,3,4,5}\right\}:\left\{10,10^{6}, 1,10^{26}, 10^{8}\right\}$, the method to choose the proper $\lambda \mathrm{s}$ is presented in [13]

$T C$ : Termination conditions and Gen $=0$ : Generation count

Output: $\bar{C}_{F}^{*}$ and $X^{*}$

1: Randomly generating $N_{P}$ strings, each of length $N_{B}$ bits to represent the individual $i\left(\left\{X_{i}\right\}\right)$

2: Dividing each string in $\left\{X_{i}\right\}$ into two substrings $\left\{\left[X_{i}^{1} X_{i}^{2}\right]\right\}, X_{i}^{1}$ has $V_{B}$ bits and $X_{i}^{2}$ has $V_{R}$ bits, letting $v_{i, n, h_{k}}=\mathrm{a} 2 \mathrm{~m}\left\{X_{i}^{1}\right\}$ and $P_{i, h_{k}}^{\mathrm{TX}}=\mathrm{b} 2 \mathrm{r}\left\{X_{i}^{2}\right\}$

3: Calculating $N_{P}$ fitness values $\bar{C}_{F}\left(v_{i, n, h_{k}}, P_{i, h_{k}}^{\mathrm{TX}}\right)$ with respect to the individual $i$

4: while $T C$ does not hold do

5: $\quad$ Gen $=$ Gen +1

6: Putting $\left\{X_{i}\right\}$ and $\bar{C}_{F}\left(v_{i, n, h_{k}}, P_{i, h_{k}}^{\mathrm{TX}}\right)$ in the mating pool for ranking

7: $\quad$ Selecting $N_{G}=N_{P} \times P_{G}$ better individuals with higher $\bar{C}_{F}\left(v_{i, n, h_{k}}, P_{i, h_{k}}^{\mathrm{TX}}\right)$ for breeding the next generation by using stochastic universal sampling operator [56]

8: Selecting a pair of parents to generate a pair of offsprings by using double point crossover operator [56]. The crossover operator is not required to be applied to all the chosen pairs, but done with a crossover probability $P_{C}$

9: $\quad$ Mutating the offsprings with a mutation probability $P_{M}$ to recover good genetic materials

10: Dividing each offspring into two sub-offsprings $\left[\left\{X_{j}^{1} X_{j}^{2}\right\}\right], X_{j}^{1}$ has $V_{B}$ bits and $X_{j}^{2}$ has $V_{R}$ bits, letting $v_{j, n, h_{k}}=\mathrm{a} 2 \mathrm{~m}\left\{X_{j}^{1}\right\}$ and $P_{j, h_{k}}^{\mathrm{TX}}=\mathrm{b} 2 \mathrm{r}\left\{X_{j}^{2}\right\}$, $j=1,2, \ldots, N_{G}$

11: Evaluating the fitness values of the offsprings, reinserting them into the present generation

12: end while

13: Finding the best fitness value $\bar{C}_{F}^{*}$ with respect to the best individual $X^{*}$ in the last generation 
TABLE II: Parameters Setting

\begin{tabular}{ll}
\hline \hline Symbols & Specifications \\
\hline \hline$N$ & 5 SUs \\
$K$ & $3 \mathrm{CUs} /$ paths/ videos \\
$\left\{H_{k}\right\}$ & $\{1,2,3\}$ \\
$W$ & $10 \mathrm{MHz}$ \\
$P^{\mathrm{M}, \mathrm{SU}}, P^{\mathrm{M}, \mathrm{RU}}$ & $10 \mathrm{~W}$ \\
$N_{0}$ & $10^{-13} \mathrm{~W}$ \\
$\eta$ & 4 (path loss exponent) \\
$\gamma_{0}$ & $5 \mathrm{~dB}$ \\
$\alpha$ & 1 \\
\hline
\end{tabular}

continuous generations and 2) Gen $=150$. In addition, the operator a $2 \mathrm{~m}$ is used to convert a binary array of the left substring into $v_{n, h_{k}}$ in the form of an $N \times H_{k}$ matrix and the operator $\mathrm{b} 2 \mathrm{r}$ is used to convert a binary array of the right sub-string into $P_{h_{k}}^{\mathrm{TX}}$ in the form of a real value [56].

Regarding the complexity of Algorithm 1, it includes memory complexity and time complexity. The memory complexity is $\mathcal{O}\left(N_{P} N_{B}\right)$, meanwhile the time complexity depends not only on $N_{P}$ and $N_{B}$ but also on the computations and the operators of GAs such as calculating fitness values, ranking, selection, crossover, and mutation. Here, the time complexity, which is caused by mutation, can be ignored because it is executed at a very low probability $\left(10^{-9}\right)$.

\section{Vi. Performance Evaluation}

\section{A. Parameters Setting}

The system parameters used for performance evaluation are listed in Table II. The distance from the MBS to the RU is fixed to $500 \mathrm{~m}$. The distances from the MBS to the SUs and from the TXs to the RXs of D2D hops in the same path are randomly uniform distributed in the ranges of $[500,1000] \mathrm{m}$ and $[1,10] \mathrm{m}$, respectively. Furthermore, the distances from the TXs of D2D hops in an arbitrary path to the SUs and to the RXs of D2D hops in the other paths are the same and randomly uniform distributed in the range of $[50,150] \mathrm{m} . P^{*}$ is set to the total transmission power of the TXs consumed in the downlink spectrum sharing without power allocation (SSO) scheme [50]. In SSO scheme, the transmission power of each TX is generated by following a random uniform distribution in the range of $[0.001,0.01] \mathrm{W}$. Then, $P^{*}$ is obtained by computing the total transmission power of all the TXs. The GAs convergence and SPO performance evaluations are presented in the sequel.

\section{B. GAs Convergence Evaluation}

We consider the first 100 generations to validate the convergence of GAs. The convergence result is shown in Fig. 2. The result demonstrates that the GAs start to converge after 50 generations when all the individuals are as good as the best one, i.e., the "Mean" fitness value of all individuals gets closer to, and then equal to the "Best" fitness value in the final generation. In addition, the "Error" becomes zero leading to the fact that all the individuals satisfy the constraints $(F=0)$. In conclusion, the GAs ensure a quick convergence rate that is able to solve the SPO problem.

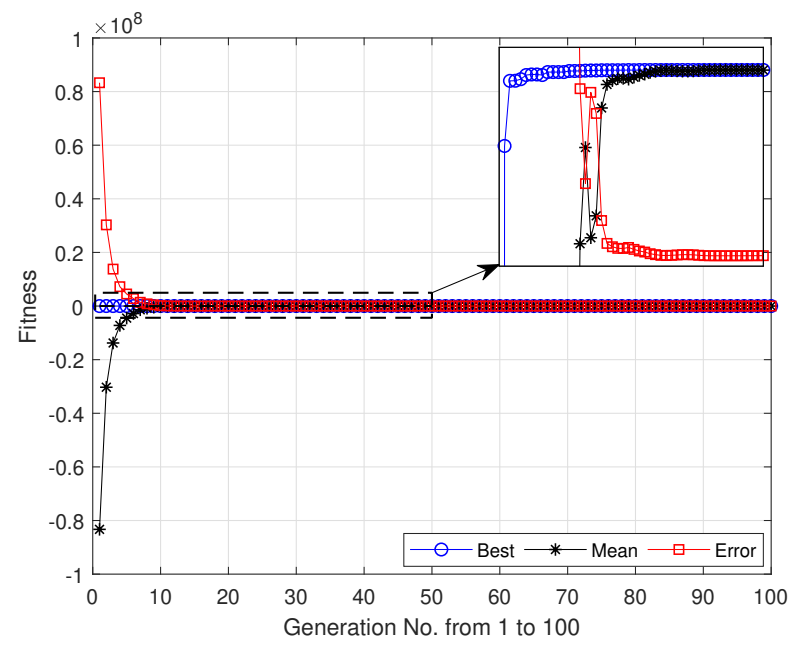

Fig. 2: Convergence rate of GAs.

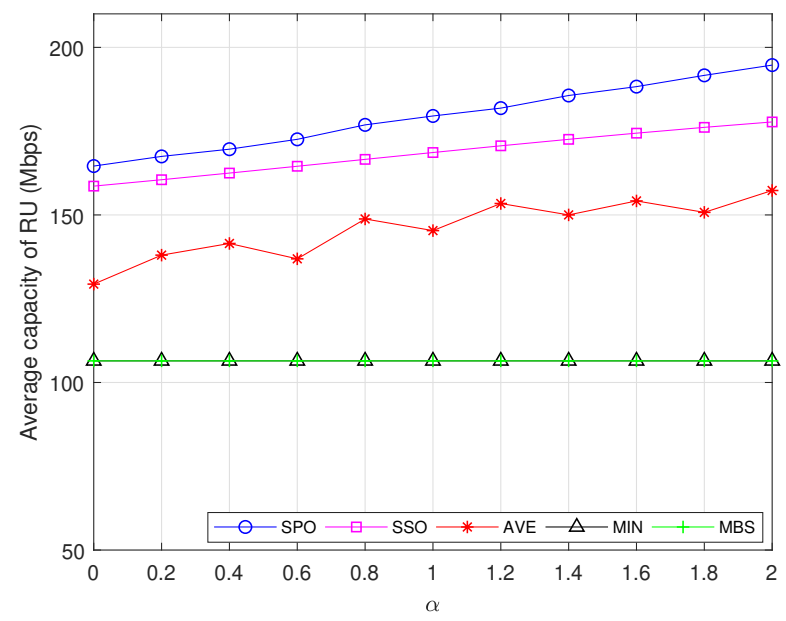

Fig. 3: Video capacity delivered to RU versus $\alpha$.

\section{SPO Evaluation}

1) Capacity Performance: To evaluate the capacity performance of the proposed SPO solution, we compare it to other benchmarks including downlink spectrum sharing without power allocation (SSO), average (AVE), minimum (MIN), and only MBS (MBS) schemes. In SSO scheme [50], only downlink spectrum sharing allocation is optimised, while the transmission powers of the TXs are fixed. In AVE and MIN schemes, we generate a large set of individuals that meet the constraints, and then compute the average value of $\bar{C}$ per individual and find the minimum value of $\bar{C}$ in the set. And in MBS scheme, the RU is served by only the MBS over common cellular communications.

The capacity performance of SPO is first evaluated by comparing with SSO, AVE, MIN, and MBS versus the exponent 


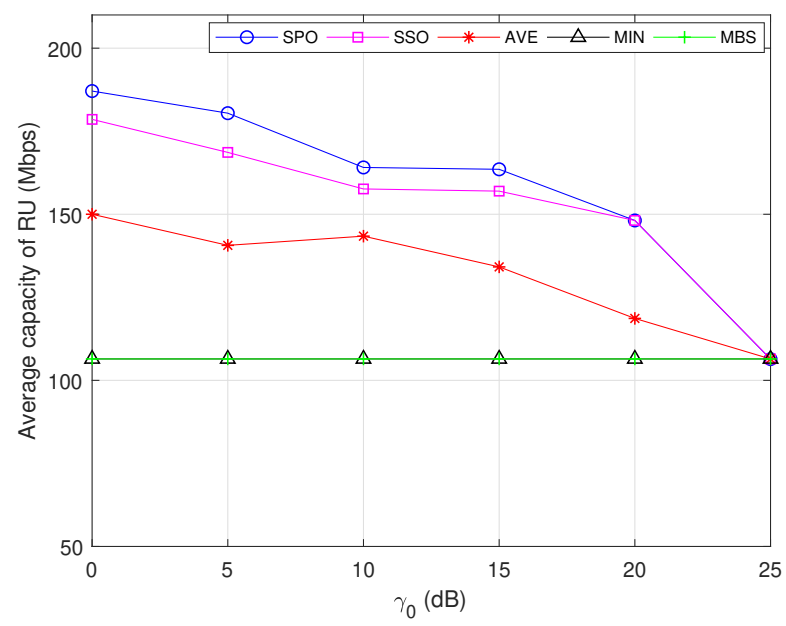

Fig. 4: Video capacity delivered to RU versus $\gamma_{0}$.

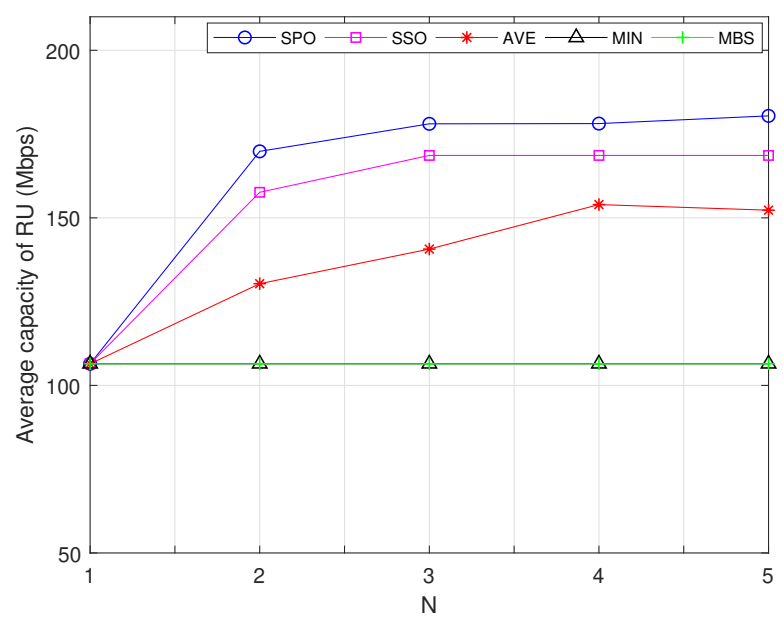

Fig. 5: Video capacity delivered to RU versus $N$.

coefficient of skewed popularity among different videos $\alpha$. As shown in Fig. 3, the diverse popularity among different videos when $\alpha$ increases leading to the result that fewer videos are much more popular than the rear ones and the optimal results focus on the few most popular videos to serve the RU better. The SPO and SSO schemes with optimal results follow the increase of $\alpha$ to serve the RU more efficiently, meanwhile the MIN and MBS schemes do not. It is obvious that the MIN scheme (the worst case of the SPO scheme) has the same result as the MBS. The AVE without optimal results cannot provide higher video delivery capacity compared to the SPO and SSO schemes. Thanks to jointly optimising downlink spectrum sharing and transmission power allocation, the SPO outperforms the other SSO, AVE, MIN, and MBS.

Next, Fig. 4 plots the capacity performance of SPO, SSO, AVE, MIN, and MBS schemes versus the target SINR $\gamma_{0}$. The

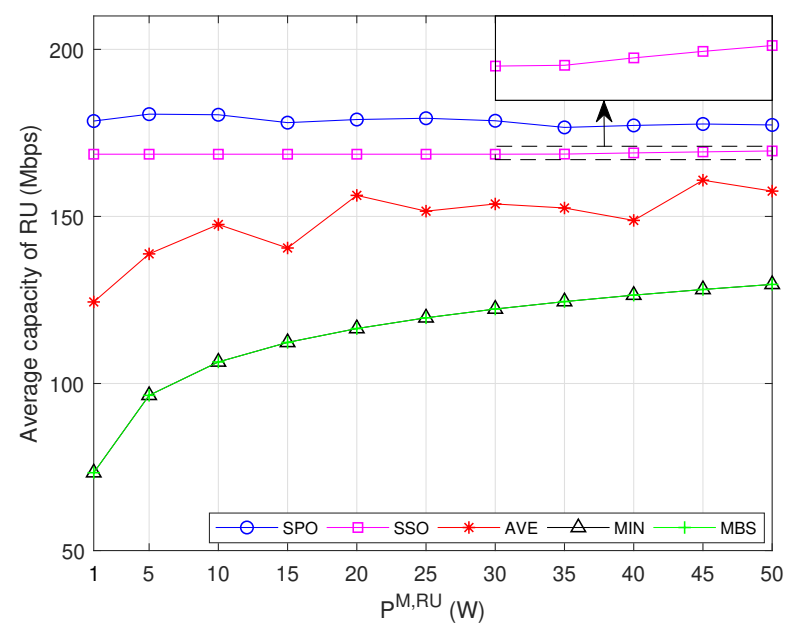

Fig. 6: System capacity delivered to RU versus $P^{M, R U}$.

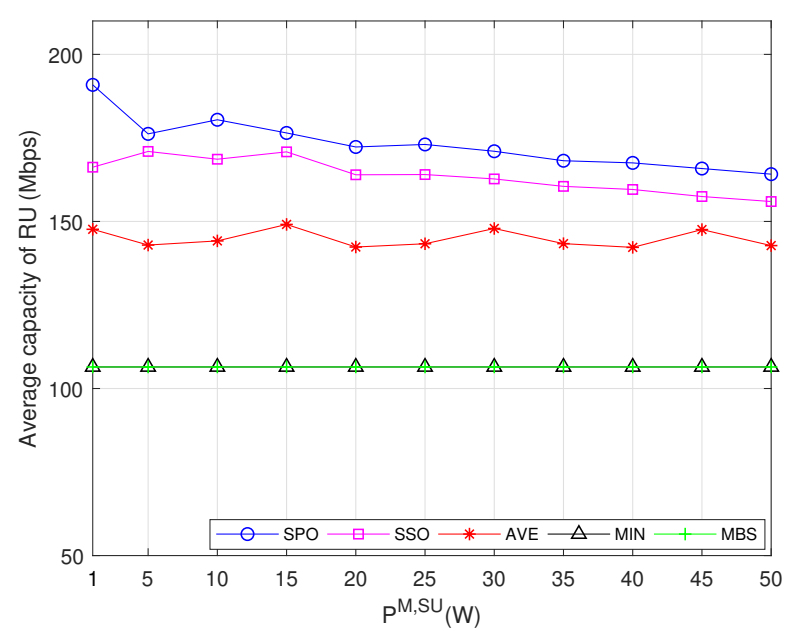

Fig. 7: System capacity delivered to RU versus $P^{M, S U}$.

increase of $\gamma_{0}$ is to guarantee the SUs higher SINR by reducing the interference impact transmitted by the TXs of D2D hops. As a result, the video delivery capacity decreases because of fewer opportunities for MHMP D2D communications. When $\gamma_{0}$ is high enough, i.e., $\gamma_{0}=25 \mathrm{~dB}$, the performance of SPO, SSO, and AVE schemes becomes that of MIN and MBS schemes since the SUs do not share any downlink spectrum resources for MHMP D2D communications. In system design, we have to carefully select a proper value of $\gamma_{0}$ such that the SPO scheme can guarantee the SUs high SINR and provide the RU with the highest video delivery capacity. It can be seen in Fig. 4 that $\gamma_{0}=5 \mathrm{~dB}$ is proper to make the SPO significantly surpass the other SSO, AVE, MIN, and MBS schemes.

Then, the capacity performance of SPO, SSO, AVE, MIN, and MBS is evaluated versus the number of SUs $N$ as shown 


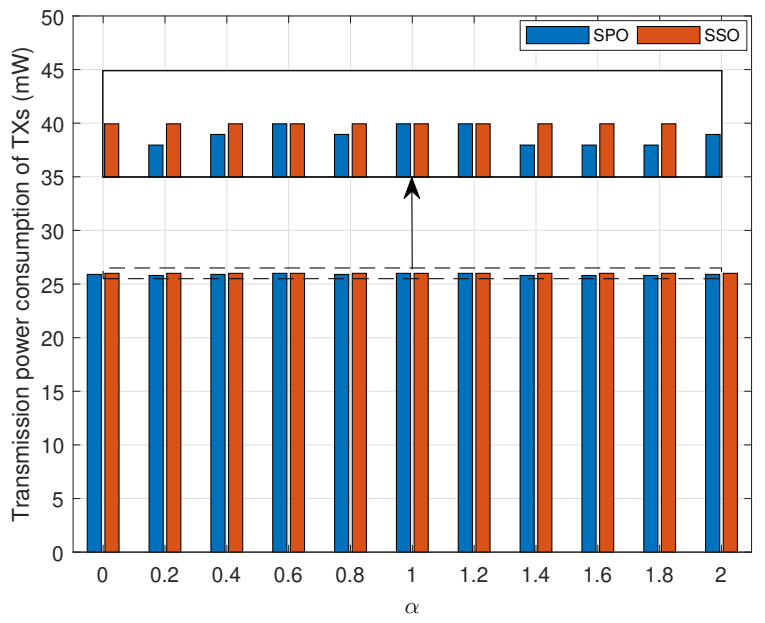

Fig. 8: Transmission power consumption of TXs versus $\alpha$.

in Fig. 5. It is clear that the more SUs the system has, the more available downlink spectrum resources the TXs reuse to establish better MHMP D2D communications. So, if $N$ is too small, i.e., $N=1$, the performance of all schemes is the same and equal to that of MIN and MBS schemes because with only one SU, MHMP D2D communications from the CUs to the RU cannot be established nor better than common cellular communications from the MBS to the RU. Increasing $N$ enables the SPO and SSO schemes to have more opportunities for downlink spectrum resource sharing to increase the video delivery capacity. However, it is noted that the video delivery capacity of SPO and SSO schemes is likely to get saturated if we continue to increase the number of SUs (e.g., $N=3$ ). The reason is that the more SUs we add to the system, the less possibility the added SUs are better than the existing ones. So, for an efficient system design, we carefully select the number of SUs such that it is enough to gain high video delivery capacity while guaranteeing a low time and memory complexity of the SPO problem. In comparison, the SPO scheme is better than the SSO scheme thanks to optimal power allocation considered, and obviously better than the other AVE, MIN, and MBS schemes.

Finally, we evaluate the capacity performance of all the schemes versus the transmission powers from the MBS to the $\mathrm{RU}\left(P^{M, R U}\right)$ and to the $\mathrm{SU}\left(P^{M, S U}\right)$ changing in the range of $[1,50] \mathrm{W}$. In Fig. 6 , the SPO scheme is not affected by $P^{M, R U}$ because the value of $P^{M, R U}$ is not high enough to make the MBS to collaborate in sending the requested videos to the RU. In other words, only MHMP D2D communications are responsible for sending all the requested videos to the RU. Similarly, the increase of $P^{M, R U}$ starts to affect the SSO scheme when it is about $35 \mathrm{~W}$, i.e., the MBS can collaborate with the SSO in sending the requested videos to the RU so as to improve the video capacity. Obviously, the increase of $P^{M, R U}$ directly makes the video capacity delivered to the RU by the MIN, MBS, and AVE schemes become higher. Meanwhile,

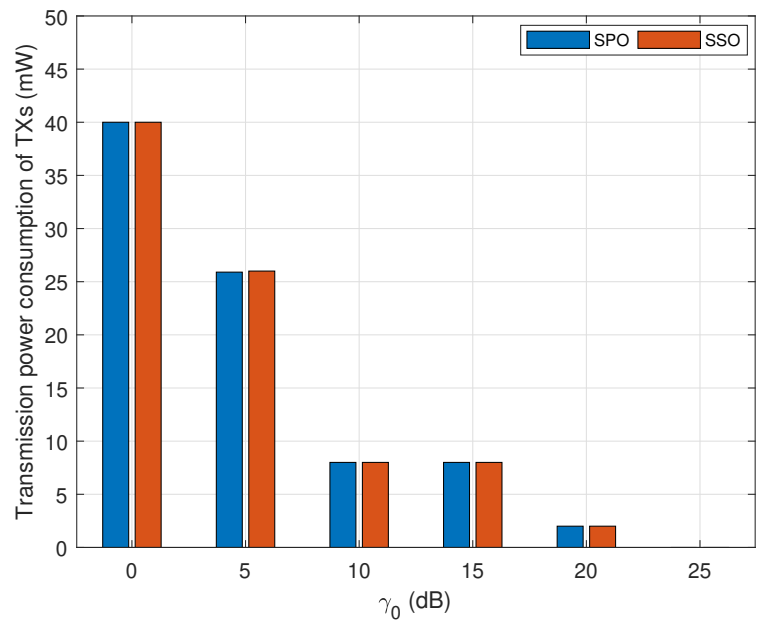

Fig. 9: Transmission power consumption of TXs versus $\gamma_{0}$.

as shown in Fig. 7, although increasing $P^{M, S U}$ yields higher opportunities for downlink spectrum resource sharing (11e), it decreases the video capacity delivered to the RU since the interference impact on the RU increases (1). It is clear that the increase of $P^{M, S U}$ does not affect the capacity performance of the MIN and MBS schemes, but slightly decreases that of the AVE scheme due to the performance reduction of the SPO scheme.

2) Power Consumption Performance: We further investigate the total transmission powers consumed by all the TXs of the SPO and SSO schemes versus $\alpha$ and $\gamma_{0}$ as shown in Fig. 8 and Fig. 9. The results show that besides providing the highest video capacity delivered to the RU as aforementioned, the SPO consumes low transmission power, even a little bit lower than the SSO scheme does (Fig. 8). The results in Fig. 8 and Fig. 9 also validate that the constraint $(11 \mathrm{~g})$ on total transmission power consumption $\left(P^{*}\right)$ always holds when solving the SPO problem to optimally allocate the transmission powers to all the TXs for MHMP D2D communications.

\section{CONCLUSION}

In this paper, we have proposed the SPO solution for video applications and services (VASs) in B5G/6G networks. The SPO solution exploits not only the storage and cached video resources but also the available downlink spectrum and energy resources of the MUs to establish the MHMP D2D communications for offloading the requested videos. The proposed SPO solution enables the RU to flexibly access the VASs from both the MBS over common cellular communications and the CUs over MHMP D2D communications. In particular, the SPO problem is formulated and solved to find the optimal downlink spectrum resources shared by the SUs and the optimal transmission powers allocated to the TXs of the D2D hops, for MHMP D2D communications. The GAs are developed to solve the SPO problem with complicated constraints and variables. Simulation results demonstrate that 
the GAs are feasible to solve the SPO problem and the SPO solution outperforms the other conventional schemes in terms of providing the highest video delivery capacity while consuming low transmission power. The paper provides the insightful analyses and discussions with respect to many system parameters and performance metrics that are helpful for designing VASs over MHMP D2D communications in B5G/6G networks.

\section{REFERENCES}

[1] Y. Hao, Y. Miao, M. Chen, H. Gharavi, and V. C. M. Leung, "6G cognitive information theory: A mailbox perspective," Big Data Cogn. Comput., vol. 5, no. 4, pp. 1-54, Oct. 2021.

[2] Cisco, "Cisco Annual Internet Report," in 2018-2023 White Paper, Mar. 2020. [Online]. Available: https://www.cisco.com

[3] M. Chen, Y. Miao, X. Jian, X. Wang, and I. Humar, "CognitiveLPWAN: Towards intelligent wireless services in hybrid low power wide area networks," IEEE Trans. Green Commun. and Networking, vol. 3, no. 2, pp. 409-417, June 2019.

[4] A. Masaracchia, L. D. Nguyen, T. Q. Duong, and M.-N. Nguyen, "An energy-efficient clustering and routing framework for disaster relief network," IEEE Access, vol. 7, pp. 56 520-56 532, Apr. 2019.

[5] M. T. Nguyen, "An energy-efficient framework for multimedia data routing in Internet of things (IoTs)," EAI Endorsed Trans. Industrial Netw. and Intell. Syst., vol. 6, no. 19, pp. 1-8, Jun. 2019.

[6] H. T. Nguyen, H. D. Tuan, T. Q. Duong, H. V. Poor, and W.-J. Hwang, "Collaborative multicast beamforming for content delivery by cacheenabled ultra dense networks," IEEE Trans. Commun., vol. 67, no. 5, pp. 3396-3406, May 2019.

[7] Q.-N. Tran, N.-S. Vo, Q.-A. Nguyen, M.-P. Bui, T.-M. Phan, V.-V. Lam, and A. Masaracchia, "D2D multi-hop multi-path communications in B5G networks: A survey on models, techniques, and applications," EAI Endorsed Trans. Industrial Netw. and Intell. Syst., vol. 7, no. 25, pp. 1-12, Jan. 2021.

[8] M. C. Erturk, S. Mukherjee, H. Ishii, and H. Arslan, "Distributions of transmit power and sinr in device-to-device networks," IEEE Commun. Lett., vol. 17, no. 2, pp. 273-276, Feb. 2012.

[9] W. Zhibo, T. Hui, and C. Nannan, "Clustering and power control for reliability improvement in device-to-device networks," in Proc. IEEE Global Commun. Conf., Atlanta, GA, Dec. 2013, pp. 573-578.

[10] Z. Zhang, R. Q. Hu, and Y. Qian, "D2D communication underlay in uplink cellular networks with distance based power control," in Proc. IEEE Inter. Commun. Conf., Kuala Lumpur, Malaysia, May 2016, pp. $1-6$.

[11] S. Maghsudi and S. Stańczak, "Hybrid centralized-distributed resource allocation for device-to-device communication underlaying cellular networks," IEEE Trans. Veh. Technol., vol. 65, no. 4, pp. 2481-2495, Apr. 2016.

[12] X. Zhang, Y. Wang, R. Sun, and D. Wang, "Clustered device-to-device caching based on file preferences," in Proc. IEEE 27th Annu. Int. Symp. Pers., Indoor Mobile Radio Commun., Valencia, Spain, Sep. 2016, pp. $1-6$.

[13] N.-S. Vo, T. Q. Duong, H. D. Tuan, and A. Kortun, "Optimal video streaming in dense $5 \mathrm{G}$ networks with D2D communications," IEEE Access, vol. 6, pp. 209-223, Oct 2017.

[14] T. L. Thanh and T. M. Hoang, "Cooperative spectrum-sharing with two-way AF relaying in the presence of direct communications," EAI Endorsed Trans. Industrial Netw. and Intell. Syst., vol. 5, no. 14, pp. 1-9, Jun. 2018

[15] M. Chen, Y. Hao, C.-F. Lai, D. Wu, Y. Li, and K. Hwang, "Opportunistic task scheduling over co-located clouds in mobile environment," IEEE Trans. Services Computing, vol. 11, no. 3, pp. 549-561, May-June 2018.
[16] T. H. Nguyen, D. Q. Nguyen, and V. D. Nguyen, "Quality of service provisioning for D2D users in heterogeneous networks," EAI Endorsed Trans. Industrial Netw. and Intell. Syst., vol. 6, no. 21, pp. 1-7, Oct. 2019.

[17] J. Song and W. Choi, "Mobility-aware content placement for deviceto-device caching systems," IEEE Trans. Wireless Commun., vol. 18, no. 7, pp. 3658-3668, July 2019.

[18] M.-P. Bui, N.-S. Vo, S. Q. Nguyen, and Q.-N. Tran, "Social-aware caching and resource sharing maximized video delivery capacity in $5 \mathrm{G}$ ultra-dense networks," Springer Mobile Netw. Appl., vol. 25, pp. 20372049, July 2019.

[19] N.-S. Vo, M.-P. Bui, P. Q. Truong, C. Yin, and A. Masaracchia, "Multitier caching and resource sharing for video streaming in 5G ultra-dense networks," IEEE Commun. Lett., vol. 24, no. 7, pp. 1500-1504, July 2020.

[20] M. Rim and C. G. Kang, "Cache partitioning and caching strategies for device-to-device caching systems," IEEE Access, vol. 9, pp. 8192-8211, Jan. 2021.

[21] Y. Fu, L. Salaün, X. Yang, W. Wen, and T. Q. S. Quek, "Caching efficiency maximization for device-to-device communication networks: A recommend to cache approach," IEEE Trans. Wireless Commun., vol. 20, no. 10, pp. 6580-6594, Oct. 2021.

[22] B. Peng, T. Peng, Z. Liu, Y. Yang, and C. Hu, "Cluster-based multicast transmission for device-to-device (D2D) communication," in Proc. IEEE Veh. Technol. Conf., Las Vegas, NV, Sep. 2013, pp. 1-5.

[23] R. Trestian, Q.-T. Vien, H. X. Nguyen, and O. Gemikonakli, "ECOM: Energy-efficient cluster-oriented multimedia delivery in a LTE D2D environment," in Proc. IEEE Inter. Commun. Conf., London, UK, 2015, pp. 1-7.

[24] H. Meshgi, D. Zhao, and R. Zheng, "Optimal resource allocation in multicast device-to-device communications underlaying LTE networks," IEEE Trans. Veh. Technol., vol. 66, no. 9, pp. 8357-8371, Sep. 2017.

[25] F. Jiang, L. Zhang, C. Sun, and Z. Yuan, "Clustering and resource allocation strategy for D2D multicast networks with machine learning approaches," China Commun., vol. 18, no. 1, pp. 196-211, Jan. 2021.

[26] M. Hmila, M. Fernandez-Veiga, M. R. Perez, and S. Herreria-Alonso, "Distributed energy efficient channel allocation in underlay multicast D2D communications," IEEE Trans. Mobile Computing, vol. Early Access, pp. 1-16, July 2020.

[27] S.-Z. Jian and T.-C. Hou, "Enabling efficient device to device multicast communication by clustering and resource sharing," in Proc. IEEE Wireless Commun. and Networking Conf., Marrakesh, Morocco, Oct. 2019, pp. 1-6.

[28] L. Yang, D. Wu, Y. Zhang, C. Yue, Y. Wu, and X. Shi, "Dynamic link selection for interference mitigation in D2D multicast content sharing networks," IEEE Commun. Lett., vol. 24, no. 1, pp. 217-221, Jan. 2020.

[29] T.-M. Phan, N.-S. Vo, M.-P. Bui, X.-K. Dang, and D.-B. Ha, "Downlink resource sharing and caching helper selection control maximized multicast video delivery capacity in dense D2D 5G networks," J. Sci. and Technol., vol. 12, no. 4.2, pp. 12-20, May 2020.

[30] N.-S. Vo, T.-M. Phan, M.-P. Bui, X.-K. Dang, N. T. Viet, and C. Yin, "Social-aware spectrum sharing and caching helper selection strategy optimized multicast video streaming in dense D2D 5G networks," IEEE Syst. J., vol. 15, no. 3, pp. 3480-3491, Sep. 2021.

[31] T.-M. Phan, N.-S. Vo, M.-P. Bui, Q.-N. Tran, H. M. Nguyen, and A. Masaracchia, "Downlink resource sharing and multi-tier caching selection maximized multicast video delivery capacity in 5G ultra-dense networks," in Proc. EAI Int. Conf. Industrial Netw. and Intell. Syst., Ha Noi, Vietnam, Aug. 2020, pp. 19-31.

[32] Q. Fu, L. Yang, B. Yu, and Y. Wu, "Extensive cooperative content caching and delivery scheme based on multicast for D2D-enabled HetNets," IEEE Access, vol. 9, pp. 40 884-40 902, Mar. 2021.

[33] Q. Li, X. Wang, D. Wang, and L. Song, "Analysis of a cooperative caching-multicast strategy in D2D-aided networks," IEEE Commun. Lett., pp. 1-5, Early Access, Apr. 2021. 
[34] M. Elnourani, S. Deshmukh, and B. Beferull-Lozano, "Distributed resource allocation in underlay multicast D2D communications," IEEE Trans. Commun., vol. 69, no. 5, pp. 3409-3422, May 2021.

[35] M. Peer, V. A. Bohara, and A. Srivastava, "Cache selection in dynamic D2D multicast networks using inhomogeneous markov model," IEEE Trans. Netw. Sci. and Engineering, vol. 7, no. 4, pp. 3235-3245, Oct.Dec. 2020.

[36] B. Ying and A. Nayak, "A power-efficient and social-aware relay selection method for multi-hop D2D communications," IEEE Commun. Lett., vol. 22, no. 7, pp. 1450-1453, Jul. 2018.

[37] J. Gui and J. Deng, "Multi-hop relay-aided underlay D2D communications for improving cellular coverage quality," IEEE Access, vol. 8, pp. 14318-14338, Jan. 2018.

[38] X. Lu, J. Zheng, C. Liu, and J. Xiao, "A mobility and activeness aware relay selection algorithm for multi-hop D2D communication underlaying cellular networks," in Proc. IEEE Inter. Commun. Conf., Paris, France, May 2017, pp. 1-6.

[39] H. Yuan, W. Guo, Y. Jin, S. Wang, and M. Ni, "Interference-aware multi-hop path selection for device-to-device communications in a cellular interference environment," IET Commun., vol. 11, no. 11, pp. 1741-1750, Mar. 2017.

[40] L. Wei, R. Q. Hu, Y. Qian, and G. Wu, "Energy efficiency and spectrum efficiency of multihop device-to-device communications underlaying cellular networks," IEEE Trans. Veh. Technol., vol. 65, no. 1, pp. 367380, Jan. 2016

[41] M.-C. Lee, M. Ji, and A. F. Molisch, "Optimal throughput-outage analysis of cache-aided wireless multi-hop D2D networks," IEEE Trans. Commun., vol. 69, no. 4, pp. 2489-2504, Apr. 2021.

[42] A. Celik, J. Tetzner, K. Sinha, and J. Matta, "5G device-to-device communication security and multipath routing solutions," Applied Netw. Sci., vol. 4, no. 102, pp. 1-24, Nov. 2019.

[43] C. Tata and M. Kadoch, "Secure multipath routing algorithm for deviceto-device communications for public safety over LTE heterogeneous networks," in Int. Conf. Future Internet of Things and Cloud, Rome, Italy, Oct. 2015, pp. 1-6.

[44] R. E. Ahmed, "A low-overhead multi-hop routing protocol for D2D communications in 5G," J. Commun., vol. 16, no. 5, pp. 1-7, May 2021.

[45] E. Arribas and V. Mancuso, "Multi-path D2D leads to satisfaction," in Int. Symp. A World of Wireless, Mobile and Multimedia Netw., Macau, China, July 2017, pp. 1-7.

[46] _ "Achieving per-flow satisfaction with multi-path D2D," Ad Hoc Netw., vol. 106, no. 1, pp. 1-16, Sep. 2020.

[47] B. Liu, Y. Cao, W. Wang, and T. Jiang, "Energy budget aware device-todevice cooperation for mobile videos," in Proc. IEEE Global Commun. Conf., San Diego, CA, Dec. 2015, pp. 1-7.

[48] Q. Wang, W. Wang, S. Jin, H. Zhu, and N. T. Zhang, "Joint coding mode and multi-path selection for video transmission in D2D-underlaid cellular network with shared relays," in Proc. IEEE Global Commun. Conf., San Diego, CA, Dec. 2015, pp. 1-6.

[49] Q.-N. Tran, N.-S. Vo, M.-P. Bui, V.-C. Phan, Z. Kaleem, and T. Q. Duong, "Resource sharing and segment allocation optimized video streaming over multi-hop multi-path in dense D2D 5G networks," in Proc. EAI Int. Conf. Industrial Netw. and Intell. Syst., Ho Chi Minh City, Vietnam, Aug. 2019, pp. 26-39.

[50] Q.-N. Tran, N.-S. Vo, T.-M. Phan, M.-P. Bui, M.-N. Nguyen, and A. Kortun, "Downlink resource allocation maximized video delivery capacity over multi-hop multi-path in dense D2D 5G networks," in Proc. Int. Conf. Recent Advances on Signal Processing, Telecommun. \& Computing, Ha Noi, Vietnam, Aug. 2020, pp. 72-76.

[51] W. Xiang, C. Zhu, C. K. Siew, Y. Xu, and M. Liu, "Forward error correction-based 2-D layered multiple description coding for errorresilient H.264 SVC video transmission," IEEE Trans. Circuits Syst. Video Technol., vol. 19, no. 12, pp. 1730-1738, Dec. 2009.

[52] X. Du, N.-S. Vo, T. Q. Duong, and L. Shu, "Joint replication density and rate allocation optimization for VoD systems over wireless mesh networks," IEEE Trans. Circuits Syst. Video Technol., vol. 23, no. 7, pp. 1260-1273, Jul. 2013.

[53] M.-P. Bui, N.-S. Vo, T.-V. Truong, T.-H. Nguyen, N. V. Nguyen, and $\mathrm{C}$. Yin, "Genetic algorithms for multi-tier caching and resource sharing optimized video streaming in 5G ultra-dense networks," in Proc. Int. Conf. Recent Advances on Signal Processing, Telecommun. \& Computing, Ha Noi, Vietnam, Aug. 2020, pp. 66-71.

[54] A. Bhardwaj and S. Agnihotri, "Energy- and spectral-efficiency tradeoff for D2D-multicasts in underlay cellular networks," IEEE Wireless Commun. Lett., vol. 7, no. 4, pp. 546-549, Aug. 2018.

[55] L. Breslau, P. Cao, L. Fan, G. Phillips, and S. Shenker, "Web caching and zipf-like distributions: Evidence and implications," in Proc. IEEE Inter. Conf. on Computer Commun., New York, NY, Mar. 1999, pp. 126-134.

[56] A. Chipperfield, P. Fleming, H. Pohlheim, and C. Fonseca, "Genetic Algorithm TOOLBOX For Using with Matlab Ver 1.2 Users Guide”. University of Sheffield, 1994.

[57] T. Fang and L. P. Chau, "GOP-based channel rate allocation using genetic algorithm for scalable video streaming over error-prone networks," IEEE Trans. Image Processing, vol. 15, no. 6, pp. 1323-1330, Jun. 2006.

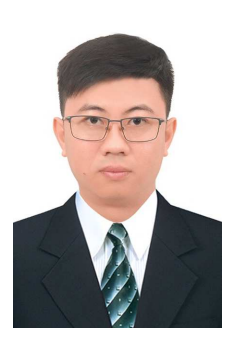

Quang-Nhat Tran received the B.S and M.Sc degrees in computer science and information technology, Mendeleev University, Moscow, Russia, in 2008. He is currently the Deputy Head of Computer Networking and IoT Department, Faculty of Information Technology, Van Lang University, Ho Chi Minh City (HCMC), Vietnam. He is also working toward the Ph.D. degree at the Institute of Fundamental and Applied Sciences, Duy Tan University, HCMC, Vietnam.

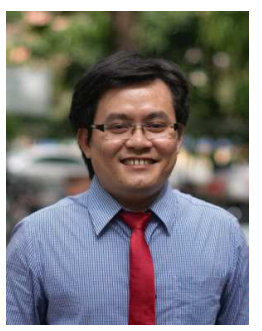

Nguyen-Son Vo received the Ph.D. degree in communication and information systems from Huazhong University of Science and Technology, China, in 2012. He is with the Institute of Fundamental and Applied Sciences, Duy Tan University, Ho Chi Minh City, Vietnam. His research interests focus on selfpowered multimedia wireless communications, quality of experience provision in wireless networks for smart cities, and IoT for disaster and environment management. He received the Best Paper Award at the IEEE Global Communications Conference 2016 and the prestigious Newton Prize 2017. He has been serving as an Associate Editor of IEEE Communications Letters since 2019; Guest Editor of Elsevier Physical Communication, Special Issue on "Mission Critical Communications and Networking for Disaster Management", 2019; Guest Editor of IET Communications, Special Issue on "Recent Advances on 5G Communications", 2018; and Guest Editor of Springer Mobile Networks \& Applications, Special Issues on "The Key Trends in B5G Technologies, Services and Applications", 2021, "Wireless Communications and Networks for 5G and Beyond", 2018 and "Wireless Communications and Networks for Smart Cities", 2017. 


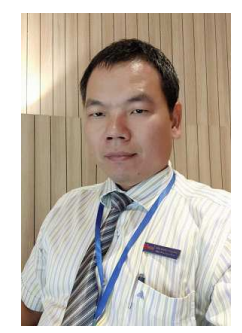

Minh-Phung Bui received the B.S. degree in information technology from Van Lang University, Ho Chi Minh City (HCMC), Vietnam, in 2000, and the M.Sc. degree in computer sciences from the University of Information Technology, HCMC, Vietnam, in 2009. He is currently the Vice Dean of Faculty of Information Technology, Van Lang University, HCMC, Vietnam. He is also working toward the Ph.D. degree at the Institute of Fundamental and Applied Sciences, Duy Tan University, HCMC, Vietnam.

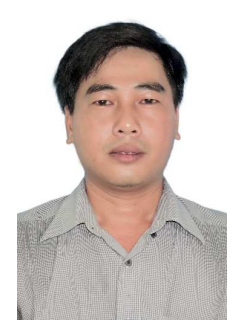

Thanh-Minh Phan received the Ph.D. degree in control engineering and automation (2021) and the M.Sc. degree in automatization (2008) from Ho Chi Minh City University of Transport, Vietnam. His research interests include wireless networked control systems, video caching and multicasting networks, and UAV communications. He is currently the Head Division of Electronic Engineering \& Telecommunications, Faculty of Electrical \& Electronics Engineering, Ho Chi Minh City University of Transport, Vietnam.

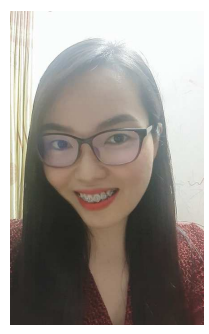

Quynh-Anh Nguyen received the M.Sc. degree in Mobile Communications from Telecom Paristech, France, in 2012, and the B.E. degree in electronics and telecommunications from Ho Chi Minh City (HCMC) University of Transport, Vietnam, in 2008. She is currently the lecturer of Electronics Engineering \& Telecommunications, Faculty of Electrical \& Electronics Engineering, HCMC University of Transport, Vietnam. She is also working toward the Ph.D. degree at the Institute of Fundamental and Applied Sciences, Duy Tan University, HCMC, Vietnam.

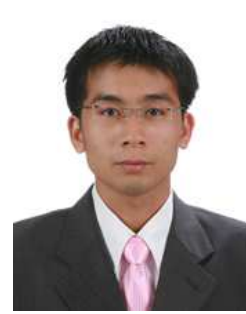

Trung Q. Duong (S'05, M'12, SM'13, F'22) is a Chair Professor of Telecommunications at Queen's University Belfast (UK), where he was a Lecturer (Assistant Professor) (2013-2017), a Reader (Associate Professor) (2018-2020), and Full Professor from August 2020. He also holds a prestigious Research Chair of Royal Academy of Engineering. His current research interests include wireless communications, machine learning, realtime optimisation, and data analytic. He is an author/co-author of over 400 publications.

Dr. Duong currently serves as an Editor for the IEEE TRANSACTIONS ON WiRELESS COMMUNiCATIONS, IEEE TRANSACTIONS ON VEHICULAR TECHNOLOGY, and an Executive Editor for IEEE COMMUNICATIONS LETTERS. He has served as an Editor/Guest Editor for IEEE TRANSACTIONS on COMMUNiCATIONS, IEEE WiRELESS COMMUNICATIONS, IEEE COMMUNICATIONS MAGAZINES, IEEE COMMUNICATIONS LETTERS, and IEEE Journal on SElEcted AREAS in COMmUnications. He was awarded the Best Paper Award at the IEEE Vehicular Technology Conference (VTCSpring) in 2013, IEEE International Conference on Communications (ICC) 2014, IEEE Global Communications Conference (GLOBECOM) 2016 and 2019, IEEE Digital Signal Processing Conference (DSP) 2017, and International Wireless Communications \& Mobile Computing Conference (IWCMC) 2019. He is the recipient of prestigious Royal Academy of Engineering Research Fellowship (2015-2020) and has won a prestigious Newton Prize 2017. He is a Fellow of IEEE (2022 Class). 